\title{
Pratiques culturales et gestion post-récolte du sorgho au Sénégal
}

\author{
Ramatoulaye FALL ${ }^{1 *}$, Mady CISSE ${ }^{2}$, Fallou SARR ${ }^{1}$, Catherine BRABET ${ }^{3}$ et \\ Eliasse DIEME ${ }^{1}$
}

\author{
${ }^{1}$ Institut de Technologie Alimentaire BP 2765 Hann-Dakar, Sénégal. \\ ${ }^{2}$ Ecole Supérieure Polytechnique BP 5085 Fann-Dakar, Sénégal. \\ ${ }^{3}$ Centre de coopération Internationale en Recherche Agronomique pour le Développement, France. \\ *Auteur correspondant ; E-mail : layetou5@yahoo.fr
}

\section{RESUME}

La non maîtrise ou l'ignorance des bonnes pratiques de stockage ou de conservation conduit souvent à des pertes des récoltes en Afrique. Ces dernières peuvent entrainer l'insécurité alimentaire qui est toujours une réalité en Afrique. Ceci illustre que les pratiques culturales et surtout la conservation post-récolte des céréales principaux aliments de base des populations, demeurent un problème majeur en Afrique. Une enquête a été réalisée au Sénégal dans les régions de Tambacounda et de Thiès pour étudier les pratiques culturales des producteurs de sorgho et caractériser la gestion post-récolte dans les zones de production. L'étude a été réalisée auprès de 384 producteurs de sorgho issus de 44 villages. Les zones ont été choisies suivant leur niveau de production et de pluviométrie. La taille de l'échantillon a été déterminée en utilisant l'approximation normale de la loi binomiale. Les résultats ont montré que dans chacune des régions, la proportion d'agriculteur masculin est de $97 \%$ et les ethnies dominantes sont les sérères $(86 \%)$ à Thiès, les peuls et les wolofs $(47 \%$ et $21 \%)$ à Tambacounda. Les semences provenaient principalement de la récolte précédente (10 à $76 \%)$ des cas et des distributeurs d'intrants agricoles (2 à $65 \%$ ). Ces taux diffèrent suivant la région. Les variétés certifiées sont cultivées par $70 \%$ des producteurs à Thiès. Les variétés traditionnelles sont utilisées par $98 \%$ de ceux de Tambacounda. Cette étude a aussi révélé que les paysans utilisent différents types de séchage à la maison ou aux champs. Parmi eux 79\% stockent leur récolte dans des cases, 14\% dans des magasins communautaires ,7\% dans des greniers sous forme de panicules ou de grains représentant respectivement 22 et $75 \%$. Ces méthodes de prise en charge post-récolte du sorgho diffèrent selon la zone et sont dans certains cas inadéquates. Elles peuvent favoriser la déperdition post-récolte du sorgho et sa contamination par les moisissures, d'où l'intérêt de bien former les acteurs de cette filière.

(C) 2020 International Formulae Group. All rights reserved.

Mots clés : Culture, stockage, céréale, enquête, variété.

\section{Cultural practices and post-harvest management of sorghum in Senegal}

\begin{abstract}
Failure to master or ignore good storage or conservation practices often leads to crop loss or spoilage of food in Africa. These can lead to food insecurity and threaten food safety. As a result, cultivation practices and especially the post-harvest conservation of cereals, the main staple food of the populations, remain a major problem in West Africa. A survey was carried out in Senegal in the regions of Tambacounda and Thies to study
\end{abstract}


the cultural practices of sorghum producers and characterize post-harvest management in the production areas. The study was carried out with 384 sorghum producers from 44 villages. The zones were chosen according to their level of production and rainfall. The sample size was determined using the normal binomial approximation. The results showed that in each of the regions, the proportion of male farmers is $97 \%$, and the dominant ethnic groups are the Serer (86\%) in Thies, the Peul and the Wolof (47\% and $21 \%)$ in Tambacounda. The seeds came mainly from the previous harvest (10 to $76 \%$ ) of the cases, and from agricultural input distributors (2 to $65 \%$ ). These rates differ by region. The certified varieties are cultivated by $70 \%$ of the producers in Thies. The traditional varieties are used by $98 \%$ of those of Tambacounda. This study also revealed that farmers use different types of drying at home or in the fields. Among them, $79 \%$ store their harvest in huts, $14 \%$ in community stores, $7 \%$ in granaries in the form of panicles or grains, representing 22 and $75 \%$ respectively. These post-harvest management methods for sorghum differ by area and are in some cases inadequate. They can encourage the postharvest loss of sorghum and its contamination by molds, hence the importance of properly training the players in this sector.

(C) 2020 International Formulae Group. All rights reserved.

Keywords: Crop, storage, cereal, survey, variety.

\section{INTRODUCTION}

En Afrique, la croissance rapide de la population fait de l'autosuffisance alimentaire un enjeu majeur des politiques agricoles. L'agriculture de subsistance est pratiquée par une majorité de petits agriculteurs. Les écarts de rendement importants et la pauvreté des sols, ainsi que d'autres contraintes telles que abiotiques et biotiques s'ajoutent à la difficulté à pratiquer une agriculture durable rentable (Macauley et Ramadjita, 2015).

Les céréales telles que le sorgho, le millet, le blé, le maïs et le riz sont des aliments de base pour la majorité de la population. En 2016 la production mondiale de sorgho était estimée à près de 64 millions de tonnes, la plaçant ainsi au cinquième rang des productions céréalières après le maïs, le blé, le riz et l'orge. Le sorgho est cultivé sur 45 millions d'hectares dans le Monde. Les principaux pays producteurs sont les EtatsUnis d'Amérique (plus de 19\% de la production mondiale), le Nigéria, le Mexique, l'Ethiopie, l'Inde, l'Argentine, la Chine et le Brésil (FAOSTAT, 2016). La part de production réservée à l'Afrique à la même année était de 30 millions de tonnes avec une superficie de 30,5 millions d'hectares cultivée, représentant respectivement $47 \%$ de la production mondiale et $69 \%$ des superficies récoltées dans le monde.

Pour disposer d'aliments tout au long de l'année, certaines méthodes traditionnelles permettant de prolonger la durée du stockage des produits ont été mises au point. Ces technologies traditionnelles généralement mises en œuvre pour la conservation des denrées alimentaires, sont souvent inadéquates avec des risques d'infestation au stockage par des insectes ravageurs de stocks où leur présence a pour résultat l'infestation de diverses moisissures toxinogènes en particulier Aspergillus (Fandohan et al., 2003 ; Gandaho et al., 2017). Ainsi, 25\% des récoltes dans le monde sont contaminées par les mycotoxines provenant des moisissures toxinogènes ; ce qui a pour conséquence la réduction de la quantité d'aliments disponibles au plan mondial (Yiannikouris et Jouany, 2002).

La production de sorgho du Sénégal en 2016 n'était que de 161000 tonnes ; ce qui est inférieur à celle de 2015 (188000 tonnes), soit une baisse de $14 \%$. Le sorgho est exploité à l'est du Bassin arachidier, au Sud dans la région de Kolda notamment dans le département de Vélingara et dans la vallée du fleuve Sénégal en culture de décrue (Infoconseil, 2006).

Dans le centre du bassin arachidier et les zones sud et est du pays, le sorgho est généralement cultivé en zone monoculture dans les bas-fonds ou en rotation avec le coton. La partie nord du sud du bassin arachidier connaît une tendance à l'extension du sol de sorgho, qui révèle une transformation des assolements et des successions culturales. 
Trois successions principales y sont identifiées sorgho-sorgho dans les bas-fonds inondés ou inondables, mil-arachide ou mil-sorghoarachide, jachère-sorgho-arachide quand la jachère couvre des surfaces importantes. Des successions triennales et quadriennales avec sorgho ont été définies dans des systèmes de culture intensifs et semi-intensifs, cependant elles ne sont pas pratiquées en parcelles paysannes (Fall et Lo, 2009).

Actuellement plus de trente variétés traditionnelles et neuf variétés certifiées sont cultivées au Sénégal. Le manque de statistiques fiables, qui demeure un problème de l'agriculture sénégalaise, empêche de disposer d'un nombre exact de producteurs de sorgho au Sénégal. L'objectif de ce travail était d'étudier les pratiques culturales et de caractériser la gestion post- récolte des producteurs de sorgho dans les régions de Thiès et de Tambacounda au Sénégal. Cette étude a été initiée dans le cadre d'une thèse de doctorat sur les contaminants du sorgho au Sénégal.

\section{MATERIEL ET METHODES \\ Choix des régions}

Les données statistiques de la production de sorgho et de la pluviométrie de 2011 à 2014 du Sénégal, fournies respectivement par la Direction de l'Analyse, de la Prévision et des Statistiques Agricoles (DAPSA) et l'Agence Nationale de l'Aviation Civile et de la Météorologie (ANACIM), nous ont permis de hiérarchiser les régions suivant ces deux paramètres : Tambacounda, Kaffrine, Kolda, Fatick, Thiès (Tableau 1).

L'analyse du Tableau 1 voudrait que les régions de Tambacounda et de Kaffrine soient choisies d'autant plus qu'elles sont complètement différentes sur le plan agro écologique et écosystémique. Un choix raisonné a permis de sélectionner les régions de Tambacounda et Thiès. Tambacounda est choisie grâce à sa position de première région productrice de sorgho au Sénégal en termes de quantité. Le deuxième choix était porté sur Thiès du fait de la forte présence de deux types de variétés de sorgho traditionnelles et améliorées, mais aussi de son agro écologie éloignée et différente de celle de Tambacounda contrairement à Kaffrine (Figure 1).

Le Tableau 2 donne les caractéristiques des deux régions enquêtées dans cette étude

\section{Conduite des enquêtes}

\section{Détermination de la taille des échantillons}

La taille des échantillons a été déterminée en utilisant l'approximation normale de la loi binomiale (Dagnelie, 1998) définie par l'équation suivante :

$\mathrm{N}=\mathrm{U}^{2} 1-\alpha / 2 \times \mathrm{P}(1-\mathrm{P}) / \mathrm{d}^{2} \quad$ (1)

$\mathrm{U}_{1-\alpha / 2}$ est la valeur de la loi normale liée à la valeur de probabilité $\alpha / 2$ avec $\alpha=5 \%$ soit $\mathrm{U}=1,96$;

$\mathrm{P}=$ proportion théorique de producteurs dans les régions ;

En optimisant le $\mathrm{P}$ avec la formule ci-dessus, $\mathrm{du}$ fait de l'indisponibilité du nombre exact de producteurs au niveau national, on obtient $\mathrm{P}=$ $50 \%$;

1-P= son complément ;

$0 \leq \mathrm{d} \leq 15 \%$, d est la marge d'erreur de l'estimation qui est fixée à $5 \%$.

En se servant de la proportionnalité des quantités de production et de la taille $\mathrm{N}$ de la population (1), nous avons effectué la répartition suivante avec la formule :

$\mathrm{Ni}=\left(\mathrm{Pi} / \sum_{\mathrm{i}=1}^{2} \mathrm{Pi}\right) \times \mathrm{N}$

$\mathrm{Ni}$ : nombre de producteurs à enquêter dans la région ;

Pi : quantité de production de sorgho de la région ;

\section{Taille et répartition de l'échantillon}

L'application de la formule 1 nous a permis d'obtenir le nombre total de producteurs à enquêter, (384). La proportionnalité suivant le niveau de production de sorgho de la région a permis de déterminer 321 producteurs dans la région de Tambacounda et 63 producteurs dans celle de Thiès.

\section{Questionnaire d'enquête}

Le questionnaire est constitué de variables qualitatives et quantitatives. Les variables qualitatives sont : provenance des semences, mode d'acquisition des parcelles exploitées, utilisation de pesticides, 
spéculations faites, variétés de sorgho cultivées, pratiques culturales. Les variables quantitatives sont : âge du producteur, nombre de parcelles utilisées par producteur et part réservée au sorgho, quantité de la production par producteur, durée de la récolte et durée du séchage.

Des entretiens individuels ont été réalisés dans toutes les localités enquêtées au niveau des départements de Thiès,
Tambacounda, Koumpentoum, par deux ingénieurs et un technicien agronome.

\section{Analyses statistiques}

Sur le plan statistique, les données de l'enquête ont été analysées sur Epi Info7.1.5.0. Les résultats ont été exprimés en fréquences et en moyennes. La comparaison des fréquences a été faite par le test de Khi2 ou ses variantes et celle des moyennes par le test t de Student. Le seuil de signification statistique était de 5\%.

Tableau 1 : Niveau de production et de pluviométrie des régions.

\begin{tabular}{lcr}
\hline \multicolumn{1}{c}{ Région } & \multicolumn{3}{c}{ Moyenne de 2011 à 2014 } \\
\cline { 2 - 4 } & Pluviométrie (mm) & Production (tonne) \\
\hline Tambacounda & 733 & 31766 \\
Kaffrine & 626 & 26025 \\
Kolda & 942 & 14377 \\
Fatick & 645 & 6304 \\
Thiès & 420 & 6248 \\
\hline
\end{tabular}

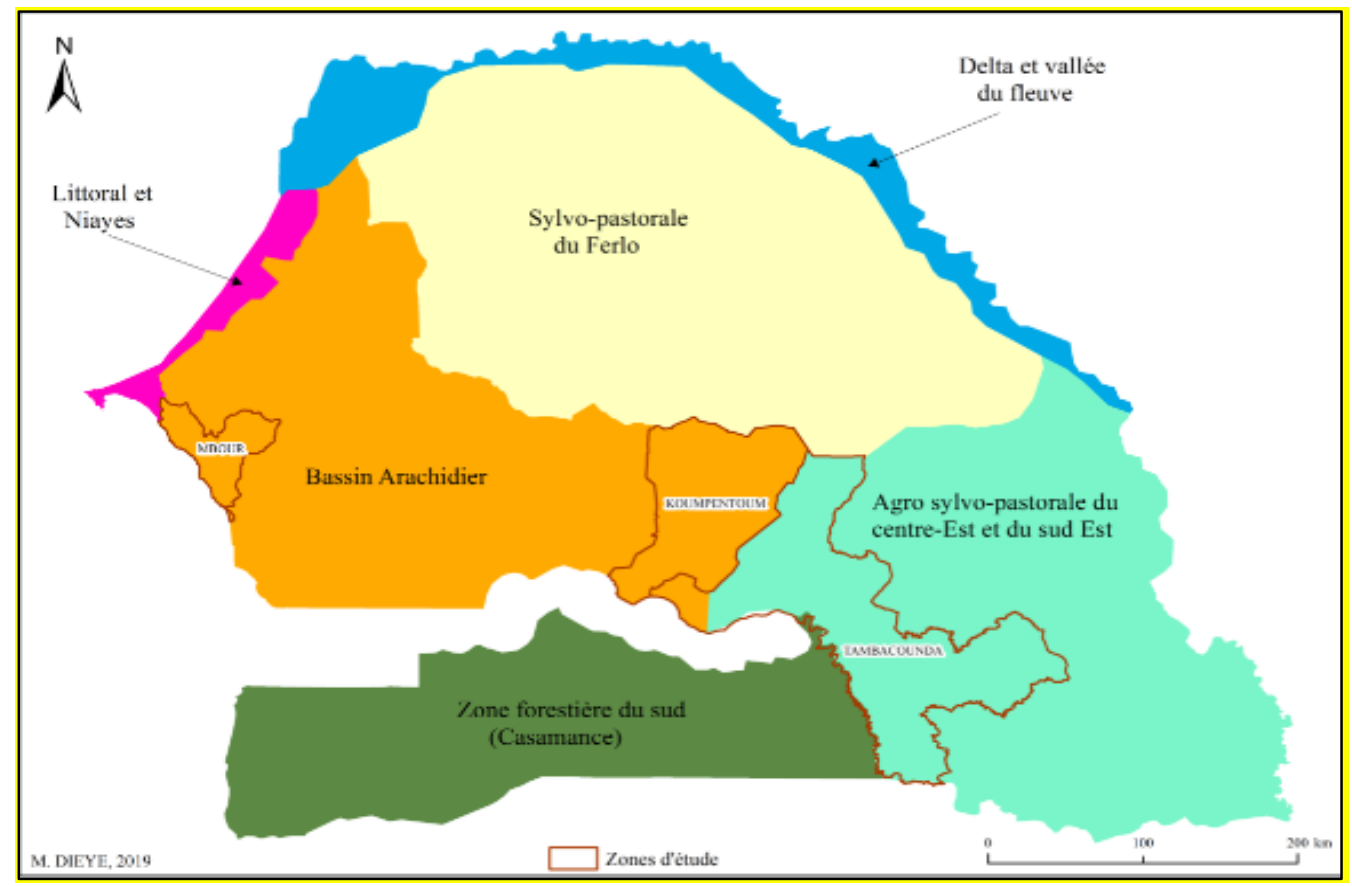

Figure 1 : Localisation de la zone d'étude dans celles agroécologiques du Sénégal. 
Tableau 2 : Caractéristiques des régions.

\begin{tabular}{lll}
\hline & \multicolumn{2}{c}{ Région } \\
\cline { 2 - 3 } & Tambacounda & Thiès \\
\hline Coordonnées géographiques & $691919,04 \mathrm{X}-1536343,06 \mathrm{Y}$ & $311025,11 \mathrm{X}-1640606,22 \mathrm{Y}$ \\
Isohyètes & $450-800 \mathrm{~mm}$ & $400-600 \mathrm{~mm}$ \\
Départements enquêtés & Tambacounda Koumpentoum & Mbour \\
\hline
\end{tabular}

\section{RESULTATS}

\section{Caractéristiques sociodémographiques des producteurs}

Parmi les éléments qualitatifs, il a été relevé ceux qui sont en rapport avec les caractéristiques sociodémographiques: le sexe, l'ethnie et la localité habitée par le producteur et un élément quantitatif qu'est l'âge moyen (Tableau 3).

La proportion des producteurs de sorgho de sexe masculin dans les deux régions est de $97 \%$ et celle des femmes de $3 \%$.

Les deux ethnies dominantes dans la région de Tambacounda sont les peuls et les wolofs respectivement avec $47 \%$ et $21 \%$ des producteurs alors que celles prépondérantes dans la région de Thiès sont les sérères et les peuls respectivement $86 \%$ et $11 \%$.

L'âge moyen des producteurs de sorgho dans les régions de Tambacounda et Thiès est respectivement de 45 et 53 ans.

\section{Production et récolte du sorgho}

Un certain nombre de matériel agricole acheté, loué ou emprunté est employé par les producteurs. La céréale est souvent cultivée en association avec d'autres spéculations (Tableau 4).

Plus de $76 \%$ des producteurs de la région de Tambacounda gardent après chaque récolte une quantité de grains servant de semences. Deux pour cent d'entre eux sont approvisionnés en semences par l'Institut Sénégalais de Recherche Agricole (ISRA).

Dans la région de Thiès par contre plus de $65 \%$ des semences sont fournies par le RESOPP contre $10 \%$ des semences qui sont issues de la récolte précédente.
Dans les deux régions le pourcentage de producteurs propriétaire de parcelles est très élevé. Il est de $81 \%$ à Thiès et plus de $93 \%$ dans la région de Tambacounda.

La plupart des personnes interrogées lors des entretiens, cultivent le sorgho en association avec d'autres spéculations que sont : l'arachide, le maïs, le mil, le niébé et des produits maraichers (Tableau 4).

La répartition des variétés traditionnelles et certifiées cultivées dans les deux régions est matérialisée dans la Figure 2. Dans la région de Tambacounda, plus de $98 \%$ des producteurs travaillent avec les variétés locales (traditionnelles) contre 1\% qui cultivent les variétés certifiées. Cette tendance est totalement inversée dans la région de Thiès avec un pourcentage de $70 \%$ de paysans utilisant les variétés certifiées contre 22\% employant les variétés locales (Figure 2).

Les quantités moyennes de la production de sorgho toutes variétés confondues dans les deux régions sont présentées dans le Tableau 5.

La quantité moyenne annuelle de la production de sorgho est de $900 \mathrm{~kg}$ dans la région de Tambacounda. Celle-ci est inférieure à celle de la région de Thiès qui est égale à $1329 \mathrm{~kg}$. La durée de récolte dans les deux régions tourne autour de 4 mois.

La main d'œuvre utilisée pour la récolte de cette céréale est diverse (Figure 3). Celle utilisée pour la culture du sorgho vient de la famille à $72 \%$ complétée si nécessaire par une main d'œuvre salariale. Celle venant de la famille et des volontaires représente $15 \%$, suivie de celle fournie par la famille et les employés $12 \%$ et enfin $1 \%$ de la main d'œuvre 
est composés de la famille, des volontaires et des employés.

\section{Gestion post-récolte et stockage du sorgho}

Le séchage du sorgho après la récolte est réalisé soit au champ soit au domicile du producteur (Tableau 6).

L'étude a montré que les proportions de producteurs qui sèchent le sorgho au champ et celles de producteurs qui le ramènent à la maison se valent $(50 \%)$. Que cela soit à la maison ou aux champs, différents types de séchage sont utilisés par les producteurs. Plus de cinquante pour cent des producteurs dans les deux régions, optent pour le séchage des panicules de sorgho à la maison au sol $(21,35 \%)$, sur bâche $(11,72 \%)$ et sur tiges de céréales $(17,19 \%)$. Concernant les 49,74\% des producteurs préférant le séchage au champ ; $16,67 \%$ d'entre eux laissent sécher directement les panicules en bottes au sol alors que les $33,07 \%$ restant choisissent d'étaler leur récolte sur des tiges de céréales (Figure 4).

Les opérations post-récoltes de battage, de vannage et d'ensachage sont réalisées par $77 \%$ des producteurs. (Tableau 6 ).

Soixante-dix-neuf pour cent des locaux de stockage sont des cases. Seuls $14 \%$ des lieux de stockage sont des magasins communautaires dans les régions de Tambacounda et de Thiès (Tableau 7). Dans le département de Mbour où le RESOPP est fortement implanté et travaille en collaboration avec les producteurs, les magasins communautaires représentent plus de $46 \%$ des lieux de stockage. Les cases (en paille ou en banco) sont faites de matériaux locaux qui sont plus disponibles et plus en adéquation avec leurs moyens financiers. Cependant, les principes de bases tels qu'une bonne aération du local, un entreposage sur palettes, un respect de la distance entre piles entre autres ne sont pas souvent respectés

Trois quarts des récoltes sont stocké sous forme de grains, $22 \%$ en panicules et $3 \%$ stockés en panicules et grains (Tableau 7).

A Tambacounda comme à Thiès, les grains de sorgho sont utilisés pour l'autoconsommation, l'alimentation du bétail, la commercialisation ou stockés comme semences (Tableau 7).

Lors de la conservation du sorgho, seuls $33 \%$ des producteurs des deux régions font recours aux fongicides (Figure 5).

Tableau 3 : Caractéristiques socio-démographiques.

\begin{tabular}{|c|c|c|c|c|c|}
\hline \multirow[t]{3}{*}{ Variable } & \multirow[t]{3}{*}{ Modalité } & \multicolumn{4}{|c|}{ Région } \\
\hline & & \multicolumn{2}{|c|}{ Thiès } & \multicolumn{2}{|c|}{ Tambacounda } \\
\hline & & Effectif & Fréquence \% & Effectif & Fréquence \% \\
\hline \multirow[t]{7}{*}{ Ethnie } & Peul & 7 & 11,11 & 152 & 47,35 \\
\hline & Wolof & 1 & 1,59 & 68 & 21,18 \\
\hline & Sérère & 54 & 85,71 & 7 & 2,18 \\
\hline & Diakhanké & 0 & 0 & 21 & 6,54 \\
\hline & Mandingue & 1 & 0 & 23 & 7,16 \\
\hline & Socé & 0 & 0 & 22 & 6,85 \\
\hline & Autres & 1 & 1,59 & 28 & 8,74 \\
\hline \multirow[t]{2}{*}{ Genre } & Masculin & 61 & 96,83 & 312 & 97,20 \\
\hline & Féminin & 2 & 3,17 & 9 & 97,20 \\
\hline Age & $\begin{array}{l}\text { Moyenne } \pm \\
\text { Ecart type }\end{array}$ & \multicolumn{2}{|c|}{$52,54 \pm 11,35$} & \multicolumn{2}{|c|}{$44,87 \pm 12,32$} \\
\hline
\end{tabular}


Tableau 4 : Production de sorgho.

\begin{tabular}{|c|c|c|c|c|c|}
\hline \multirow[t]{2}{*}{ Variable } & \multirow[t]{2}{*}{ Modalité } & \multicolumn{2}{|c|}{ Thiès } & \multicolumn{2}{|c|}{ Tambacounda } \\
\hline & & Effectif & Fréquence \% & Effectif & Fréquence \% \\
\hline \multirow{6}{*}{$\begin{array}{l}\text { Provenance des } \\
\text { semences }\end{array}$} & ISRA/RESOPP & 41 & 65,08 & 6 & 1,87 \\
\hline & Récolte & & 23,81 & & \\
\hline & Achat & 15 & 1,59 & 246 & 76,64 \\
\hline & Achat/voisin & 1 & & & \\
\hline & & 6 & 9,52 & 33 & 10,28 \\
\hline & & & & 36 & 11,21 \\
\hline \multirow{4}{*}{$\begin{array}{l}\text { Matériel } \\
\text { agricole }\end{array}$} & Appartient au & 8 & 12,70 & 15 & 4,67 \\
\hline & producteur & & & & \\
\hline & Location ou emprunt & & & & \\
\hline & & 55 & 87,30 & 306 & 95,33 \\
\hline \multirow{6}{*}{ Type de matériel } & & 25 & 39,68 & 67 & 20,87 \\
\hline & Semoir/Houe sine & & & & \\
\hline & Semoir/Houe & 11 & 17,46 & 74 & 23,05 \\
\hline & sine/charrette & & & & \\
\hline & Autres & & & & \\
\hline & & 27 & 42,86 & 180 & 56,07 \\
\hline \multirow{6}{*}{$\begin{array}{l}\text { Spéculations } \\
\text { faites autre que } \\
\text { le sorgho }\end{array}$} & Mil-maïs-arachide & 1 & 1,59 & 136 & 42,37 \\
\hline & $\begin{array}{l}\text { Mil-maïs-arachide- } \\
\text { niébé }\end{array}$ & & & & \\
\hline & Mil- maïs & 4 & 6,34 & 40 & 12,46 \\
\hline & Autres & & & & \\
\hline & & 8 & 12,70 & 9 & 2,80 \\
\hline & & 50 & 79,37 & 139 & 42,37 \\
\hline \multirow[t]{2}{*}{ Parcelle utilisée } & & 51 & 80,95 & 300 & 93,45 \\
\hline & Appartenance & & & & \\
\hline
\end{tabular}

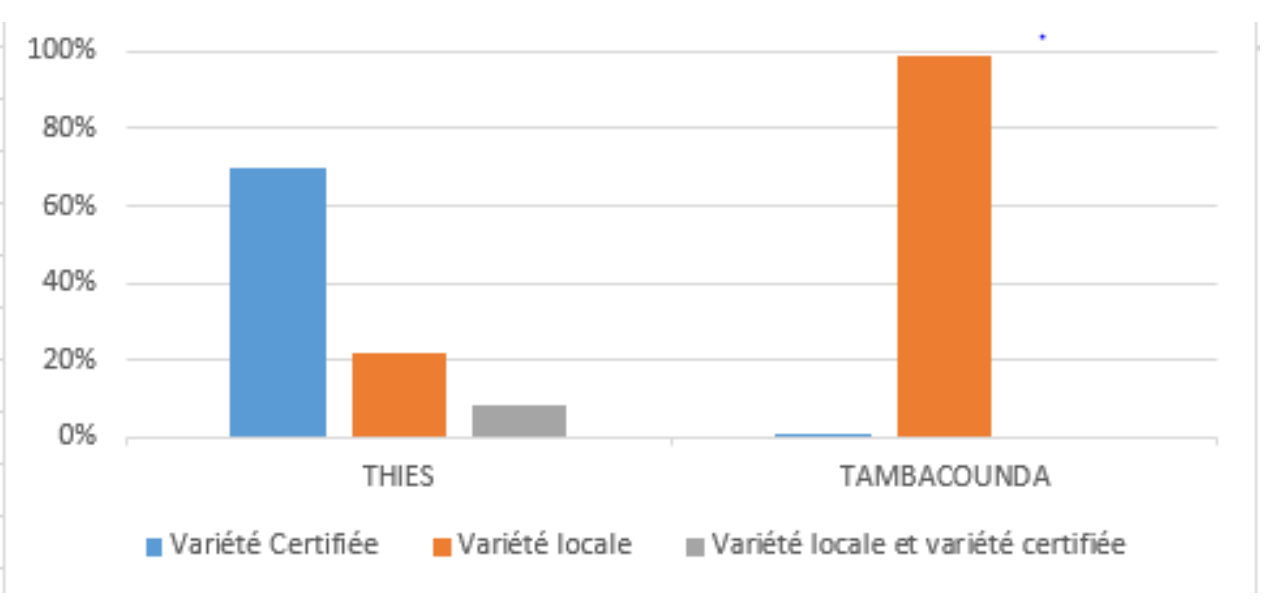

Figure 2 : Répartition des variétés de sorgho dans les 2 régions. 
Tableau 5 : Quantité de production de sorgho par producteur et durée de récolte.

\begin{tabular}{lllllll}
\hline Région & $\begin{array}{l}\text { Quantité } \\
\text { moyenne de } \\
\text { production }(\mathbf{k g})\end{array}$ & Min-Max & $\begin{array}{l}\text { Ecart- } \\
\text { type }\end{array}$ & $\begin{array}{l}\text { Durée de } \\
\text { production } \\
\text { (Mois) }\end{array}$ & $\begin{array}{l}\text { Min- } \\
\text { Max }\end{array}$ & $\begin{array}{l}\text { Ecart- } \\
\text { Type }\end{array}$ \\
\hline Tambacounda & 900 & $50-6250$ & 859 & 4,08 & $3-7$ & 0,76 \\
Thiès & 1329 & $100-5000$ & 947 & 3,84 & $3-7$ & 0,83 \\
\hline
\end{tabular}

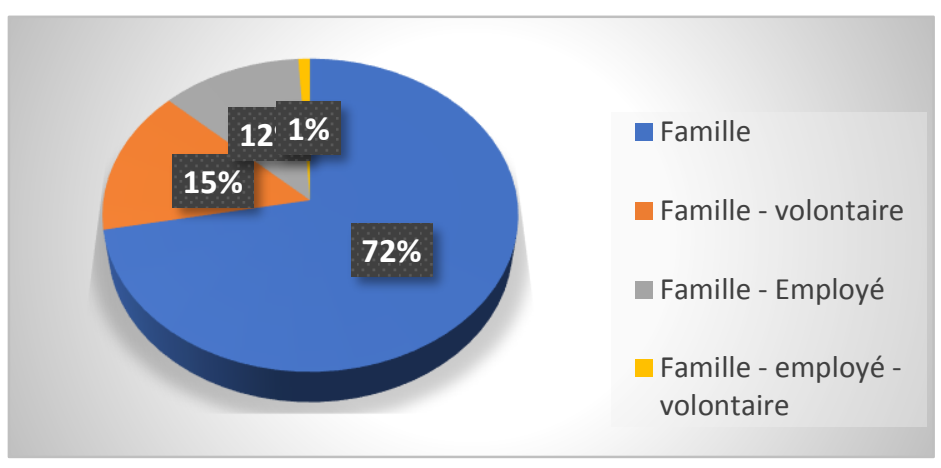

Figure 3 : Répartition de la main d'œuvre lors de la récolte de sorgho.

Tableau 6 : Gestion post-récolte du sorgho.

\begin{tabular}{llcc}
\hline Variable & Réponse & Effectif & Fréquence (\%) \\
\hline $\begin{array}{l}\text { Transport de la récolte au lieu de } \\
\text { stockage }\end{array}$ & Véhicule & 7 & 1,82 \\
& Charette & 377 & 98,18 \\
& & & \\
\hline Mode séchage & Séchage au champ & 191 & 49,74 \\
& Séchage à la maison & 193 & 50,26 \\
& & & \\
\hline \multirow{2}{*}{ Opérations effectuées } & Battage-Vannage- & 295 & 76,82 \\
& Ensachage & & \\
& Pas de battage & 82 & 21,35 \\
& Battage-Ensachage & 7 & 1,83 \\
\hline
\end{tabular}

Panicules en bottes au sol à la maison Sur bâche à la maison

Sur tiges de céréales à la maison

Panicules en bottes au sol aux champs

Sur tiges de céréales aux champs

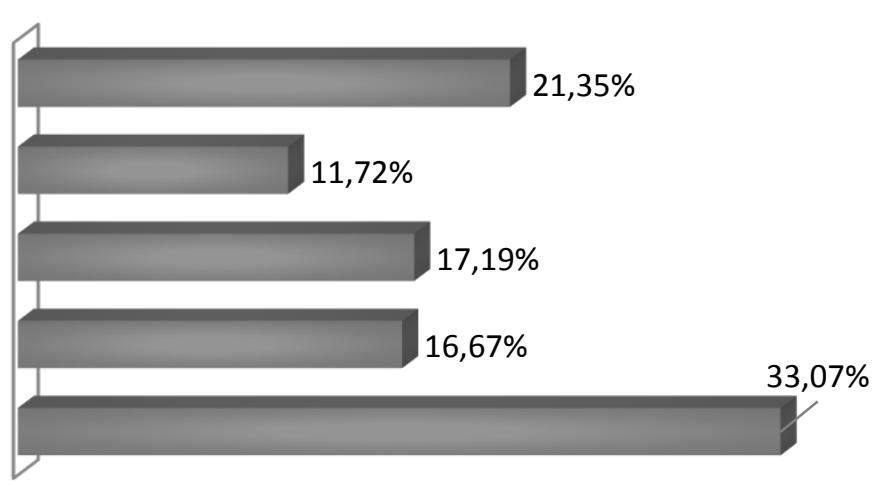

Figure 4 : Différents types de séchage du sorgho dans les deux régions. 
Tableau 7 : Stockage du sorgho.

\begin{tabular}{|c|c|c|c|}
\hline Variable & Réponse & Effectif & Fréquence $(\%)$ \\
\hline $\begin{array}{l}\text { Type de } \\
\text { stockage }\end{array}$ & $\begin{array}{l}\text { Case } \\
\text { Magasin communautaire } \\
\text { Grenier }\end{array}$ & $\begin{array}{l}304 \\
52 \\
28\end{array}$ & $\begin{array}{l}79,16 \\
13,54 \\
7,30\end{array}$ \\
\hline $\begin{array}{l}\text { Mode de } \\
\text { stockage }\end{array}$ & $\begin{array}{l}\text { Grains } \\
\text { Panicules } \\
\text { Grains-Panicules }\end{array}$ & $\begin{array}{l}287 \\
85 \\
12\end{array}$ & $\begin{array}{l}74,74 \\
22,14 \\
3,13\end{array}$ \\
\hline \multirow{4}{*}{$\begin{array}{l}\text { Utilisation } \\
\text { sorgho }\end{array}$} & Autoconsommation-Semences & 89 & 23,18 \\
\hline & $\begin{array}{l}\text { Autoconsommation-Aliment de bétail-Semences } \\
\text { Autoconsommation-Aliment de bétail- } \\
\text { Commercialisation-Semences }\end{array}$ & 79 & $\begin{array}{l}20,57 \\
18,75\end{array}$ \\
\hline & $\begin{array}{l}\text { Autoconsommation-Commercialisation-Semences } \\
\text { Autoconsommation-Commercialisation }\end{array}$ & 49 & 12,76 \\
\hline & Autres & $\begin{array}{l}32 \\
63\end{array}$ & $\begin{array}{l}8,33 \\
16,41\end{array}$ \\
\hline
\end{tabular}

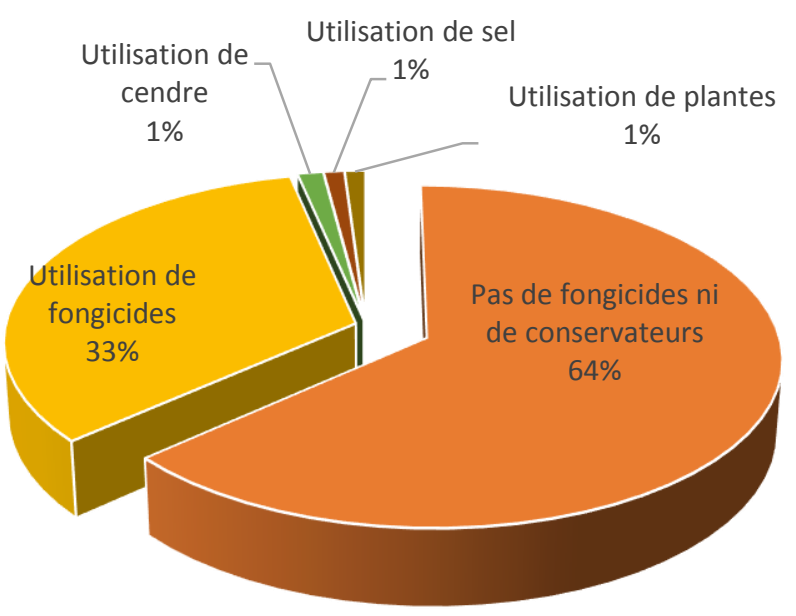

Figure 5 : Répartition des producteurs utilisant de fongicides. 


\section{DISCUSSION}

Ce diagnostic couvrant deux régions du Sénégal, productrices de sorgho a permis de mettre en évidence les pratiques liées au stockage du sorgho et de situer le niveau de technicité des producteurs quant à la conservation de leur récolte.

Les hommes constituent $97 \%$ des chefs d'exploitation, producteurs du bassin arachidier du Sénégal et les 3\% restants sont des femmes (Sarr, 2013). Ceci est en harmonie avec nos résultats de l'enquête sur le genre des producteurs de sorgho dans les régions de Thiès et de Tambacounda. Cette répartition des producteurs est incluse dans les tendances nationales rapportées par l'ANSD sur «le Recensement Général de la Population et de l'Habitat, de l'Agriculture et de l'Elevage (ANSD, 2013) », qui donne un taux de 88,7\% de producteurs masculins au Sénégal contre $11,3 \%$ de femmes en milieu rural.

Une étude sur la production de sorgho parmi les petites exploitations agricoles au Kenya, a montré que les semences de sorgho provenaient des agriculteurs $(33,7 \%)$, du voisin ou du marché $(24,2 \%)$ et des distributeurs d'intrants agricoles $(42,1 \%)$ (Kange et al., 2014). Ces résultats sont en harmonie avec ceux de notre étude dans la région de Thiès, qui ont montré que la plus grosse part des semences provenait des distributeurs d'intrants (RESOPP). La RESOPP est une structure de multiplication de semences professionnelles. Toutes les variétés de sorgho distribuées par le RESOPP sont homologuées et certifiées par l'Institut Sénégalais de Recherche Agricole (ISRA).

Une partie importante des producteurs n'est pas propriétaire des outils agricoles disposés. Cependant le matériel utilisé est pour la plupart manuel. Celui à traction animale regroupe les houes sine, houes occidentales, les semoirs, souleveuses et les charrues. Cependant, dans les trois départements, on note une quasi-absence d'équipements motorisés (batteuse, tracteurs, etc.). La culture et la récolte restent par conséquent manuelles avec l'utilisation des animaux de traits (ânes, chevaux, bœufs). Cette situation rejoint celle notée par Sarr (2013) dans le bassin arachidier du Sénégal dans le cas de la production de l'arachide.

L'enquête montre que le sorgho est cultivé en association avec d'autres spéculations telles que l'arachide, le maïs, le mil, le niébé et des produits maraichers. La culture d'autres spéculations en association avec le sorgho permet l'utilisation des champs en rotation. Ceci participe à la sauvegarde et la fertilité des sols. Elle assure également une diversité de produits (céréales, légumineuse, produits maraichers) aux producteurs pour la consommation et favorise également une augmentation des revenus de ces derniers. Dans une étude sur le sorgho au Bénin, il a été montré que la grande majorité des producteurs enquêtés cultivent séparément les différentes variétés de sorgho dans des champs, avec $52,81 \%$ en culture pure (sans association avec d'autres cultures) et $32,58 \%$ en association avec d'autres cultures (maïs, le mil, niébé, arachide, etc.) (Missihoun et al., 2012).

Les différents types de séchage utilisés dans les deux régions (étalage sur tiges de céréales aux champs, à la maison, directement sur le sol aux champs, sur une bâche à la maison ou champs) restent intéressant, à partir du moment où la durée et les conditions de séchage permettent une bonne conservation des grains ou panicules. Ces conditions ne sont pas toujours respectées avec l'impatience de certains producteurs lors du séchage. Dans certaines conditions de température et d'humidité, le développement des moisissures lié dès fois à la présence des insectes ravageurs sur les grains est très propice. L'action des moisissures modifie le pouvoir germinatif et les qualités organoleptiques et alimentaires des grains (Hamé, 2013). Plusieurs chercheurs ont montré différentes courbes décroissantes de 
séchage exprimant l'évolution de la teneur en eau en fonction du temps (Belghit et al., 2000 ; Kouhila, 2001 ; Gandaho et al., 2017).

D'après Kange et al. (2014), au Kenya les grains de sorgho sont soit stockés sous forme décortiquée $(88,4 \%)$ ou en panicules $(11,6 \%)$. Dans notre étude, les deux principaux modes de stockage en panicules et en grains sont pratiqués dans les régions de Thiès et de Tambacounda. Le stockage en grains reste majoritaire avec près de $75 \%$ des producteurs.

Certains effectuent le battage juste après le séchage et d'autres gardent les panicules un moment avant battage ou bien ne l'effectuent pas du tout avant utilisation, car disent-ils la panicule offre une certaine protection aux grains de sorgho contre les attaques d'insectes et de moisissures. Ces deux modes de stockage sont donc liés à l'environnement et au contexte local et pourraient être améliorés en y associant d'autres méthodes plus modernes et ayant montré leurs preuves.

Lors de la conservation du sorgho, seuls $33 \%$ des producteurs des deux régions font recours aux pesticides. Les traitements à base de pesticides souvent en poudres s'effectuent à mains nues et sans protection respiratoire, sans tenir compte des risques. Selon l'Organisation Mondiale de la Santé, chaque année, les pesticides causent accidentellement quelques 20000 décès et près de 750000 personnes souffrent de leurs effets chroniques spécifiques ou non, principalement dans les pays en développement (Gueye et al., 2012). L'utilisation des produits phytosanitaires non maîtrisée à cause du manque de formation peut être une source de nuisances pour la santé humaine et pour l'environnement (Kanda, 2013; Ngakiama et al., 2019). Bien que l'utilisation de pesticides puisse être un problème de santé publique, il n'en demeure pas moins qu'elle est parfois nécessaire pour une bonne conservation des céréales. Cependant l'usage de produits naturels à la place de ceux chimiques est préférable mais n'est toujours pas le cas chez les agriculteurs. Dans une étude au Niger, il a été rapporté que $8,8 \%$ des producteurs utilisaient des substances inertes (sable fin et centre) (Hamé et al., 2013).

\section{Conclusion}

Cette étude, dont l'objectif était de caractériser les pratiques culturales et la gestion post- récolte des producteurs de sorgho dans les régions de Thiès et de Tambacounda, met en exergue plusieurs points cruciaux de la filière sorgho au Sénégal. Il s'agit entre autres de la domination masculine dans la culture du sorgho, et de trois ethnies (Peul, Wolof, Sérère) dans la zone d'intervention. La production se fait avec des semences d'origines différentes, qui sont des variétés traditionnelles ou certifiées suivant la région. Le choix de stocker la récolte dans des cases ou grenier après les opérations de séchage, de battage (ou pas) et vannage incombe au producteur, et dépend de ses moyens matériels ou financiers. L'enquête révèle qu'une partie importante des producteurs fait recours aux fongicides pour mieux conserver le sorgho. Il faut savoir que des mauvaises conditions de séchage ou de stockage peuvent favoriser des pertes ou des rendements moindres de sorgho, mais également générer des attaques d'insectes et de moisissures nuisibles à la santé. Ainsi une formation des acteurs de la filière sorgho sur les bonnes pratiques de récolte, de séchage et de stockage devient une nécessité. Une étude consistant à évaluer l'efficacité des opérations post-récoltes d'une part et à identifier les contaminants potentiels du sorgho d'autre part avec mesure systématique de tous les paramètres, permettrait d'améliorer les revenus des producteurs mais surtout contribuer à l'atteinte de la sécurité alimentaire et sanitaire au Sénégal.

\section{CONFLIT D'INTERETS}

Les auteurs déclarent qu'il n'existe aucun conflit d'intérêts. 


\section{CONTRIBUTIONS DES AUTEURS}

La collecte des données a été réalisée par RF et FS; l'analyse des données et la rédaction du présent document ont été faites par RF, MC, CB et ED.

\section{REMERCIEMENTS}

Nous remercions l'Institut de Technologie Alimentaire (ITA), le Programme de Productivité Agricole en Afrique de l'Ouest (PPAAO/WAAP), le Projet d'Appui à la Promotion des Enseignantes-chercheuses du Sénégal (PAPES) ainsi que tous les acteurs et producteurs de sorgho pour leur soutien et appui.

\section{REFERENCES}

ANSD. 2013. Recensement Général de la Population et de l'Habitat, de l'Agriculture et de l'Elevage (RGPHAE), rapport définitif. Sénégal; $30 \mathrm{p}$.

Belghit A, Kouhila M, Boutaleb BC. 2000. Experimental Study of Drying Kinetics by Forced Convection of Aromatic Plants. Energy Conversion and Management, 41(12) : 1303-1321.

DOI: 10.1016/S0196-8904(99)00162-4

Dagnelie P. 1998. Statistique Théorique et Appliquée (Tome 1). De Boeck et Larcier : Bruxelles.

Fall AA, Lo M. 2009. Etude de références sur les céréales : mil, sorgho, maïs et fonio au Sénégal. Etude de référence sur les céréales : mil, sorgho, maïs et fonio au Sénégal. Rapport Coraf/Wecard 2009. https://issuu.com/coraf/docs/rapportfinal etudedebasesenegal/12

Fandohan P, Hell K, Marasas WFO, Wingfield MJ. 2003. Infection of maize by Fusarium species and contamination with fumonisin. In africa. African Journal of Biotechnology, 2(12): 570-579. DOI: https://doi.org/10.5897/AJB2003.0001110.
FAO (Food and Agriculture Organization of the United Nations). 2016. FAOSTAT Available : http://www.fao.org/faostat/fr/\#data/QC

Gandaho S, Goudjinou C, Tchobo FP, Soumanou MM. 2017. Séchage du sorgho produit dans le Nord-Bénin : Caractérisation thermophysique et qualité microbiologique $\mathrm{du}$ produit séché. Journal of Applied Biosciences, 112: 11016-11024. DOI: 10.4314/jab.v112i1.6 Gueye MT, Seck D, Wathelet JP, Lognay G. 2012. Typologie des systèmes de stockage et de conservation du maïs dans l'est et le sud du Sénégal. BASE, 16(1): 49-58.

https://popups.uliege.be:443/17804507/index.php?id=8402.

Hamé AK, Aboubacar K, Ousmane ZM, Abdou MB. 2013. Connaissances paysannes sur la conservation du sorgho et du mil dans le département d'Aguié au Niger. Journal des Sciences de l'Environnement, 2(1): 10-18.

https://www.researchgate.net/publication /279912734

Infoconseil. 2006. Etat des lieux de la filière céréales locales au Sénégal, Dakar, Sénégal, Gret, Enda graf, SNC Lavalin, Cintech, MAE, CDE, ACDI, MIA, 54p.

Kanda M, Djaneye-Boundjou G, Wala K, Gnandi K, Batawila K, Sanni A and Akpagana K. 2013. Application des pesticides en agriculture maraichère au Togo. VertigO - la revue électronique en sciences de l'environnement, 13(1) : 117. DOI : $10.4000 /$ vertigo.13456.

Kange AM, Cheruiyot EK, Ogendo JO, Arama PF, Ochola SO. 2014. Pre-and postharvest factors affecting sorghum production (Sorghum bicolor L. Moench) among smallholder farming communities. International Journal of Agronomy and Agricultural Research (IJAAR), $\quad$ 5(4): $\quad 40-47$. 
http://www.innspub.net/wp-

content/uploads/2014/10/IJAAR-

V5No4-p40-47.pdf

Kouhila M. 2001. Étude expérimentale et théorique de cinétiques de séchage convectif partiellement solaire des plantes médicinales et aromatiques (Menthe, Verveine, Sauge et Eucalyptus) de la région de Marrakech. Thèse de Doctorat d'État. Université Cadi Ayyad, Marrakech: p 1-170.

Macauley H, Ramadjita T. 2015. Les cultures céréalières : riz, maïs, millet, sorgho et blé. Africa Rice Center, Benin. 38p.

Missihoun AA, Agbangla C, AdoukonouSagbadja H, Ahanhanzo C, Vodouhe R. 2012. International Journal of Biological and Chemical Sciences, 6(3): 1003-1018. DOI:

http://dx.doi.org/10.4314/ijbcs.v6i3.8
Ngakiama GN, Mbela GK, Pole CS, Kyela CM, Komanda JA. 2019. Analyse des connaissances, attitudes et pratiques des maraîchers de la Ville de Kinshasa en rapport avec l'utilisation des pesticides et l'impact sur la santé humaine et sur l'environnement. International Journal of Innovation and Applied Studies, 26 (2): 345-356. http://www.ijias.issrjournals.org/abstract.php?article=IJIAS18-348-04.

Sarr S. 2013. Mécanisation agricole et productivité des filières céréalières : cas $\mathrm{du}$ bassin, arachidier. Mémoire de fin d'étude à l'Université de Thiès (ENSA) ; $75 \mathrm{p}$.

Yiannikouris A, Jouany J-P. 2002. Les mycotoxines dans les aliments des ruminants, leur devenir et leurs effets chez l'animal. INRA Prod. Anim., 15(1): 3-16. 